\title{
Determination of the Neck Size between Powders during Sintering Process Using Finite Element Methods
}

\author{
Anas Obeed Balod ${ }^{1}$, Ziad Shakeeb Al Sarraf ${ }^{2}$, Anas Abid Mattie ${ }^{3}$ \\ ${ }^{1}$ Department of Mechanical Engineering, University of Mosul, Mosul, IRAQ, anasobeed@uomosul.edu.iq \\ ${ }^{2}$ Department of Mechanical Engineering, University of Mosul, Mosul, IRAQ, ziadalsarraf@uomosul.edu.iq \\ ${ }^{3}$ Duhok Technical Institute, Duhok Polytechnic University, Kurdistan Region, IRAQ, Anasmattie@dpu.edu.krd
}

\begin{abstract}
Today, sintering considers one of the significant process that can be use in powder technology to produce a new solid product from powders using thermal energy. Many parameters can be successfully controlled by this process such as temperature, Particle size, process time, structure geometry, powder density and powder composition. Study and analysis the behaviour of powder during sintering process was carried out using finite element methods. The simulation provides two styles of discrete method and Qusi-static method. This research contributes two types of processes in order to simulate the copper powder during sintering process and to determine the variation through using contact and shrinkage ratios of powder behaviours. Finally, a comparison between the two styles of discrete element method explains how the selected parameters were impact on sintering process.
\end{abstract}

Keywords: discrete element method, powder composition, process parameters

Received: August 22, 2020 / Accepted: December 05, 2020 / Online: December 10, 2020

\section{INTRODUCTION}

This template, modified Sintering can be known as the thermal treatment of a powder, which its temperature always below the melting point of the main constituent, for the purpose of bonding between particles by increase its strength. Powder technology become one of the important technologies that can be utilized in many applications, which the technology has many steps, one of theses important steps is sintering process which is recommended in operation due to high facility and ability to overcome many issues. Understanding the whole process of sintering is required to solve errors that initiated experimentally during diffusion between powders [1]. In this work, discrete element method was performed as a numerical method to represent the mechanism of sintering process through covering of two styles: dynamics rigid bodies based on Newton's second law and the second style which use model without dynamics based on Qusi-static. Many studies are carried out on study, analysis and simulation of sintering process using finite difference method between powders under select different parameters and process conditions. Part of these studies is concerned analytically to simulate the sintering process of powders using two types of diffusions with grain boundaries and surface conditions, the analytical results are agreed with numerical one for neck size and shrinkage ratios
[2]. A simulation study on model of the densification between powders, type spherical is extracting behaviour between powders, using discrete element method. The study focuses on surface, size, boundary diffusion, applied force, and angle which the results are confirmed analytically, showing that the angle type dihedral significantly affects on neck size ratio [3], [4]. Other studies are carried out numerically to simulate the sintering process and to determine the behaviour due to re arrangement of powders, which concludes the effectiveness of contact between powders [5], however studying the effect of temperature and force are covered experimentally [6], [7]. A viscoelastic model for solid state sintering process is simulated using discrete element methods, the simulation is done under different materials, which the numerical data are found to be more closed to the experimental data within the influence of viscoelastic [8]. Investigation the influence of heat rate, thickness, density and rearrange of powder, is revealed through simulation of multilayer composite during sintering process. It is seen that the electrode which is performed in study, exhibit low in discontinuity along with increased heat rate, however, the rearrangement of powder enhanced due to electrode connectivity [9]. Further, the contact type of powder is seen clearly through a simulation study on powders using discrete element method, which the simulation is carried out on bimodal, the result 
confirms tat the connectivity is directly influence by size and type of contact [10]. Finally, this research presents simulation models of sintering process based on discrete element method. The successful models were carried out between aluminium powders using two styles of discrete element method, which the behaviour of styles for powders during sintering process were identified for the selecting discrete and Qusi-static method.

\section{PARTICLE ANALYTICAL FORMULA FOR SINTERING PROCESS}

Discrete models of sintering process are considered an interaction of particles and describing the located problems at particle necks. In fact, sintering is treated as the adhesion processes which is activated thermally and produces contact between particles. In 1958, Coble proposed sintering process to develop a first spherical model between particles of grain boundary diffusion, then the model was corrected and its equation was extracted by Coblenz et al as shown [10], [11].

$$
\frac{a}{R}=\left(\frac{192 t}{\tau_{g}}\right)^{1 / 6}
$$

While, the second equation represents the shear stress as shown:

$$
\tau_{g}=\frac{K T R^{4}}{\Omega \delta_{g} D_{g} \gamma_{s}}
$$

The solution of sintering process were extracted analytically, which the results were theoretically collected using approximation factors in mechanism of diffusion flow of particle and its grain geometry. However, the model has limitation in involving all factors that is observed experimentally. Unlike real model of sintering process has more than one mechanism, the predicted models cannot combine multiple mechanism.

\section{NUMERICAL MODELS OF SINTERING WITH DEM}

The study and analysis of sintering processes were successfully done using discrete method, which the method is applied with two different styles in order to extract the behaviour of powders. The models of powders were designed and simulated according to the spherical shape of particles, which the geometry of particles allow the simulation program to show the contact between them and analyzed the effect of touch one particle to another particle. In 1971, Cundall [12] presented a theoretical concept of discrete element method (DEM). The (DEM) is relatively new numerical method based on the discrete model of material, which can be used to extract many dynamical parameters such as forces, displacements and acceleration for a number of particles that totally contain powders. The method has two different styles to determine the sintering process between aluminium powders. Materials can be represented as rigid or deformable when it analyzed by discrete element method, which the contact forces exist due to materials interaction. Different effect such as elasticity, friction, cohesive and adhesive, damping and viscosity can be included in modeling by DEM, which allow the method to be an efficient tool and useful for modeling system under different conditions.

\section{THE VISCOUS MODEL OF SINTERING FOR DYNAMICS AND QUSI-STATIC METHODS}

Discrete Element Method (DEM) was implemented to verify from suggested models in this study. Then the numerical technique was performed to calculate the motion of large number of particles and analyzed their different scale sizes. Nowadays, DEM covered wide range of processes and has ability to overcome many engineering problems, especially those related to the granular and discontinuous materials. This gave more advantageous to the method to be used and applied very close to molecular dynamics. Although, the initiated of the technique is conducted to study the mechanics of rock, but now it applied effectively to model micro structure. Based on Newton's law, the movement of solid particles is defined as follows.

$$
m_{i} A=F_{i}
$$

where $m_{i}$ mass of the particle, $F_{i}$ represent the force summation, which these forces are divided into external force $\left(F_{e x}\right)$ and contact force $\left(F_{i j c}\right)$, initiated between particles. The equation of forces was written below as follows:

$$
F_{i}=F_{e x}+\sum_{j=1}^{n_{i}} F_{i j c}
$$

The numerical model was created between two particles. The particles were assumed to have free sintering, and then the bonding of particles was characterized. It can be seen here that the developed model was implemented by previous studies that carried out using discrete element method [13], [14], [15]. The governing equation for the normal forces resistance and sintering forces) contact between two spherical particles is derived according to the same radius (R), as shown below.

$F_{n}=F_{v}+F_{s}$

Where $F_{v}$ is viscous force, while $F_{s}$ is sintering force. Both forces have mathematical expression as mentioned below in Eq. (6) and Eq. (7) respectively.

$$
F_{v}=\frac{\pi a^{4}}{8 D_{b}} V_{n}
$$


Where $a$ the radius of the particle grain boundary, $D_{b}$ is the diffusion coefficient and $V_{n}$ is the relative velocity in normal direction [15].

$$
F_{s}=\pi \gamma s\left[4 R\left(1-\cos \frac{\Psi}{2}\right)+a \sin \frac{\Psi}{2}\right]
$$

Where $\psi$ is the dihedral angle, ${ }^{\gamma S}$ is the surface energy. The geometrical parameters of the two contact particles were shown in Fig.1. it was mentioned here within the figure, that for favourable rearrangement of particles, the tangential force was taken into account. A thermo-viscoelastic model is also introduced along to the extension of viscous model. Fig. 2 shows the presented rheological model through adding of elastic part to enrich the material properties and characterised it during sintering.

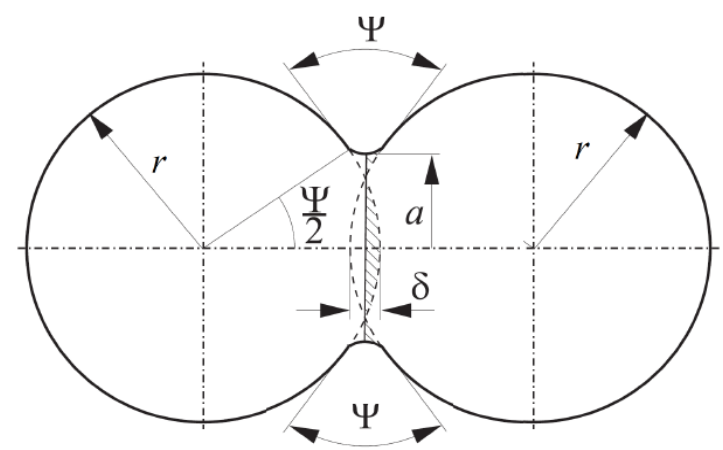

Fig. 1. Geometry of sintering for two particles contact [15]

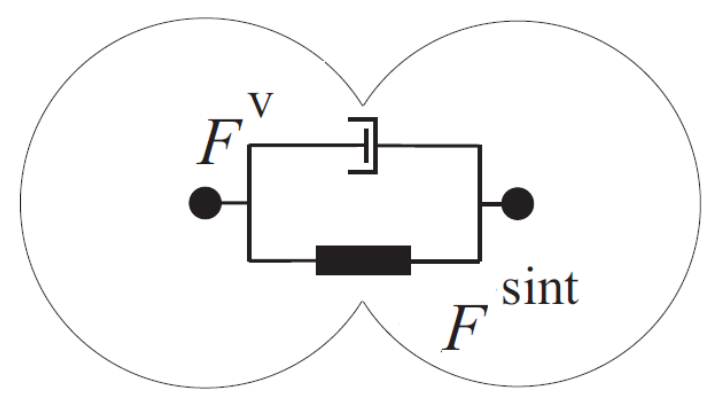

Fig. 2. Fig. 2 Rheological graph of viscous free sintering model [15]

A constitutive model of sintering process is directly depending on certain diffusion mechanism, which the values determined from the mechanism of grain boundary. The diffusion coefficient $D_{b}$ between particles can be expressed in mathematical expression as follows in equation (8). Coble's presented a model to control the initiation of neck radius of overlap between contact particles, as shown in equation (9). While the maximum value of sintering condition of contact particles at neck size can be mathematically express as seen in equation (10). At end, governing equation to determine the effective radius due to a different size along with change in radius is expressed mathematically by equation (11), [15].

$$
\begin{aligned}
& D_{b}=\frac{\Omega}{K T} \delta_{b} D_{b} \cdot e^{\left(\frac{-Q_{b}}{R T}\right)} \\
& \dot{a}=-\left(R V_{n} / a\right)
\end{aligned}
$$

$$
a_{m}=\frac{R \sin \psi}{2}
$$

$R_{e f f}=\frac{2 R_{i} R_{j}}{R_{i}+R_{j}}$

The second method that has been implemented in this study is Qusi-static method, which is performed successfully in discrete element to simulate sintering models between particles. Many studies have been incorporated in the dynamical formulation in the discrete element methods [3]. Parhami et al. present a concept of dynamical simulation, using Qusai-static method to implement the lattice type of discrete method. In this model, the center of particle is considered by a node, and the connection between any two neighbouring particles was carried out through apply discrete element method [10], [14]. Fig. 3 shows the two dimensional of two powders connected together in a small position which known neck [13]

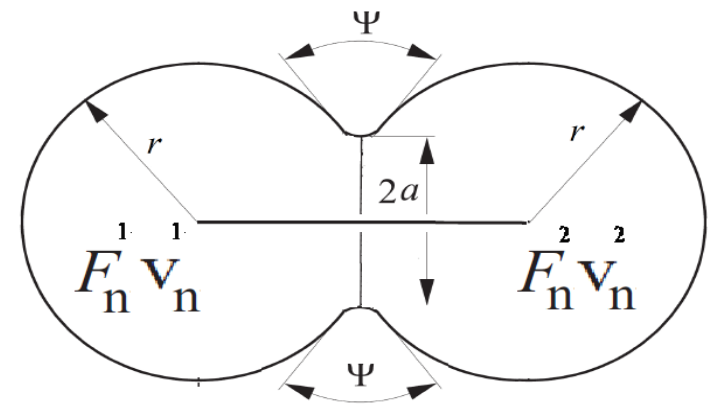

Fig. 3. Model of two particles, using Qusi-static model [13]

The relative axial velocity between two contact particles can be described by analysis the rate set up of one particle relative to another one. A proposed formula for calculating of axial velocity can be written below [13].

$V_{n}=\frac{8 D_{b}}{a^{2}}\left[\sigma+\gamma s K(r)-\frac{2 \gamma}{a} \sin \frac{\psi}{2}\right]$

Following the previous expression, the summation of curvature $\mathrm{K}(\mathrm{a})$ for the principal free surface at the edge of the grain boundary and is given by expression: 
$K(a)=-\frac{1}{s}+\frac{1}{a} \sin \frac{\Psi}{2}$

A constant model of two bonding particles by neck part with radius of surface curvature is suggested by Coble [10]. The model is carried out through intersect spheres joined by neck segments, result to make dihedral angle $\Psi$ [3]. When $r>a>$ $\mathrm{s}$, to preserve volume to first order, the expression can be written as shown below:

$\frac{1}{S}=\frac{4 R\left[1-\cos \frac{\Psi}{2}\right]}{a^{2}}$

By substituting the last two equations (14) and (13), into equation of equations (12)

$V_{n}=\frac{8 D_{b}}{a^{2}} \sigma-\frac{8 D_{b} \gamma s}{a^{4}}\left[4 R\left(1-\cos \frac{\psi}{2}\right)+a \sin \frac{\psi}{2}\right]$

Where normal stress on the inside particle grain boundary is expressed by $\sigma=\left(F_{n} / \pi a^{2}\right)$. Finally, substituting the expression of normal stress into equation (15) leads to obtain the equation (16) of normal force initiated between two contact particles during sintering process.

$$
F_{n}=\frac{\pi a^{4}}{8 D_{\text {eff }}} V_{n}+\pi \gamma_{s}\left[4 R\left(1-\cos \frac{\psi}{2}\right)+a \sin \frac{\psi}{2}\right]
$$

\section{MATERIAL PARAMETERS SELECTION}

In this study, the copper have been selected to be the material of powders that used in sintering process. The simulation program was ready to run after build up material modal, in addition to complete other requirements modals. Table I shows the mechanical and thermal data obtained from copper.

TABLE I. MECHANICAL AND THERMAL PROPERTIES OF COPPER

\begin{tabular}{ll}
\hline \multicolumn{2}{c}{ Properties } \\
\hline Density (gr./cm3) & 8.700 \\
Young's Modulus (MPa) & 118 \\
Melting Point, $(\mathrm{K})$ & 1358 \\
Poisson's Ratio & 0.31 \\
Fracture Toughness, $(\mathrm{MPa} / \mathrm{m})$ & 7.2 \\
Flexural Strength, $(\mathrm{MPa})$ & 350 \\
Hardness Vickers, $(\mathrm{kGmm}-2)$ & 320 \\
Thermal expansion
\end{tabular}

Thermal expansion coefficient,
The type of sintering process is varied from one powder materials to another one, which the type of material has significant impact on process behaviour. Table II contributes the input data of copper that implemented in simulation processing models for sintering.

TABLE II. INPUT PARAMETERS FOR COPPER MATERIAL USED IN SIMULATION PROCESS

\begin{tabular}{ll}
\hline \multicolumn{1}{c}{ Properties } & \multicolumn{1}{c}{ Copper } \\
\hline Specific surface energy $\gamma \mathrm{S}$ & $1.72 \mathrm{~J} / \mathrm{m} 2$ \\
Specific grain-boundary energy $\gamma \mathrm{g}$ & $1 \mathrm{~J} / \mathrm{m} 2$ \\
Grain-boundary thickness pre-exponential grain- & $5.12 \times 10-15 \mathrm{~m} 3 / \mathrm{s}$ \\
boundary diffusion coefficient $\delta g$ Dgo & \\
Gas constant Rr & $8.3144621 \mathrm{~J} /(\mathrm{mol} \mathrm{K})$ \\
Boltzmann's constant, k & $1.3806503 \times 10-$ \\
& $23 \mathrm{~m} 2 \mathrm{~kg} /(\mathrm{s} 2 \mathrm{~K})$ \\
Initial particle radius R & $22.5 \times 10-6 \mathrm{~m}$ \\
Atomic volume $\Omega$ & $1.18 \times 10-29 \mathrm{~m} 3$ \\
Dihedral angle $\Psi$ & $146^{\circ}$ \\
Sintering temperature T & $1027^{\circ} \mathrm{C}$ \\
Activation energy for grain boundary diffusion & $1.05 \times 105 \mathrm{~J} / \mathrm{mol}$ \\
Qg & \\
\hline
\end{tabular}

\section{SimUlation AND COMPUTER PROGRAMMING OF FREE SINTERING PROCESSES}

This study was aimed to perform numerical methods by simulating different process of powders based on discrete element method. The simulation of sintering models was developed to study the influence input parameters between two particles during process such as surface curvature, grain boundary and surface diffusion for particles. Other simulation steps were prepared prior to start simulation such as mesh modal, part assignment, contact modal, material modal, specify position ... etc. Two styles of discrete element method are carried out using program code to describe the sintering process and to solve the undeniable parameters between contact particles. Which steps of their programs can be shown in the Fig. 4. within a figure, the out put of the finite difference code contributes sintering parameters such as the radii at the neck between contact particles, the shrinkage ratio and the change in surface curvature during process.

$(10-6 / \mathrm{K})$ 


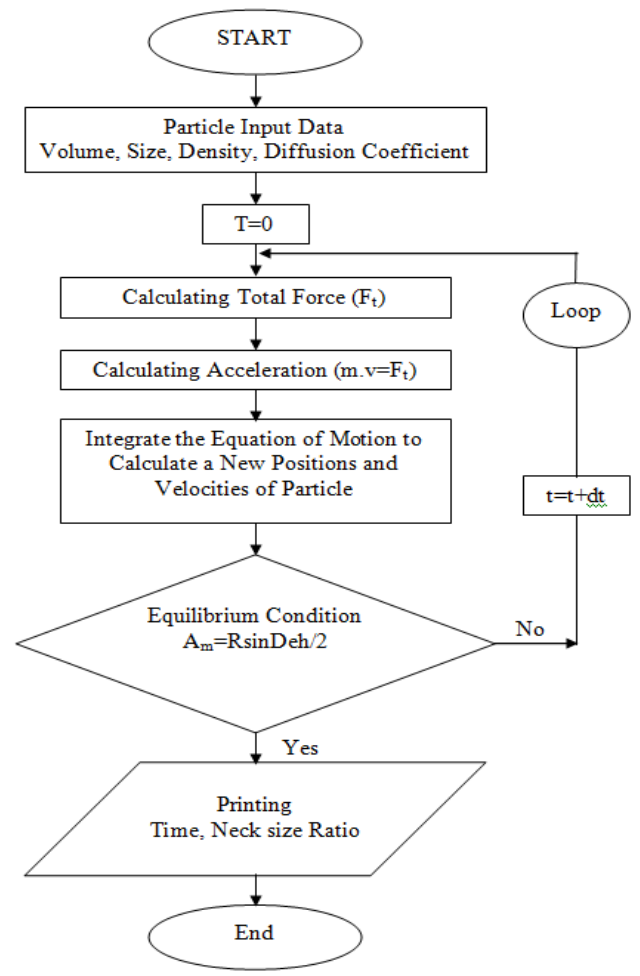

(a)

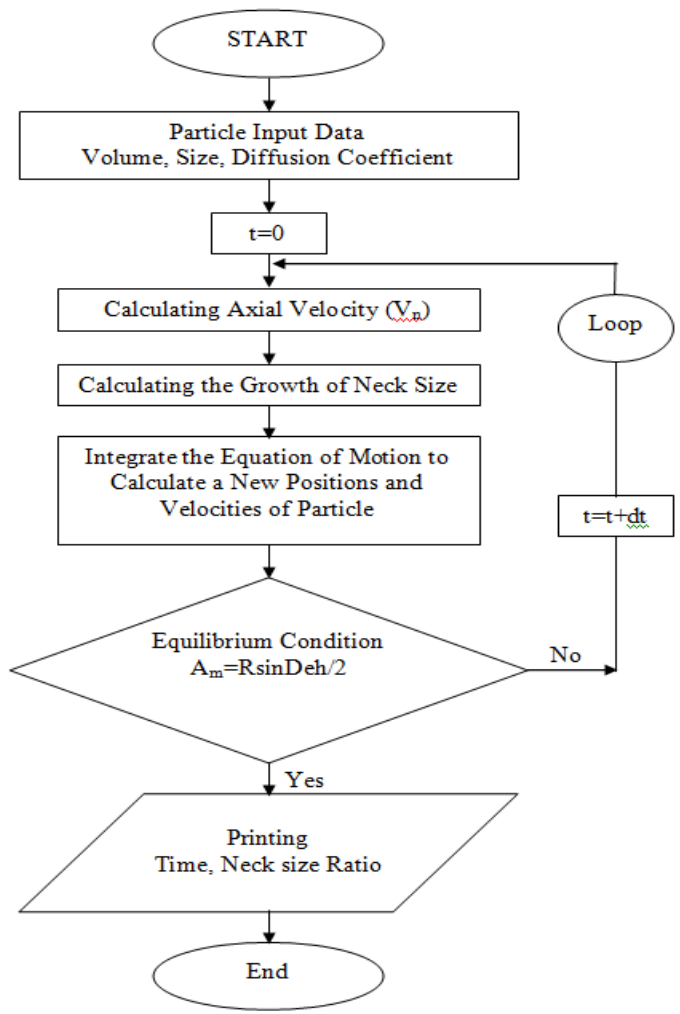

(b)

Fig. 4. Schematic diagrams for the discrete element method (DEM): (a) The first style of DEM, dynamics method (b) The second style of DEM, Qusistatic method

\section{RESULTS AND DISCUSSIONS}

In this study, the simulation of sintering process was carried out under the condition of free sintering process, which means that the effect of stress is equal zero. Copper powders were used in numerical simulation, and parameters of these powders were used as input value in computer program. The viscous model has been used, and this model contains overlap between two sphere powders. Two styles of discrete element method have been used in the numerical simulation such as dynamics and Qusi-static method. Neck size ratio plays an important role in sintering process because we can know sintering stages depends on this factor, and this factor equal the neck radius to powder radius. Fig. 5 shows the neck size ratio as a function of the sintering time for sintering between two copper powders, and this result has been achieved using the numerical model which represents Qusi-static method of discrete element method. It can be seen here that the neck ratio significantly increases with increasing sintering time and reaches maximum value up to 0.9 during consumed time 430 hr. Also, the curve indicates a rapid transformation of the sintering stages, which means that initial stage exist fast rate compared with final stage of process. In addition, the increased ratio of neck size significantly influenced by grain boundary velocity and grain boundary diffusion due to rise in temperature.

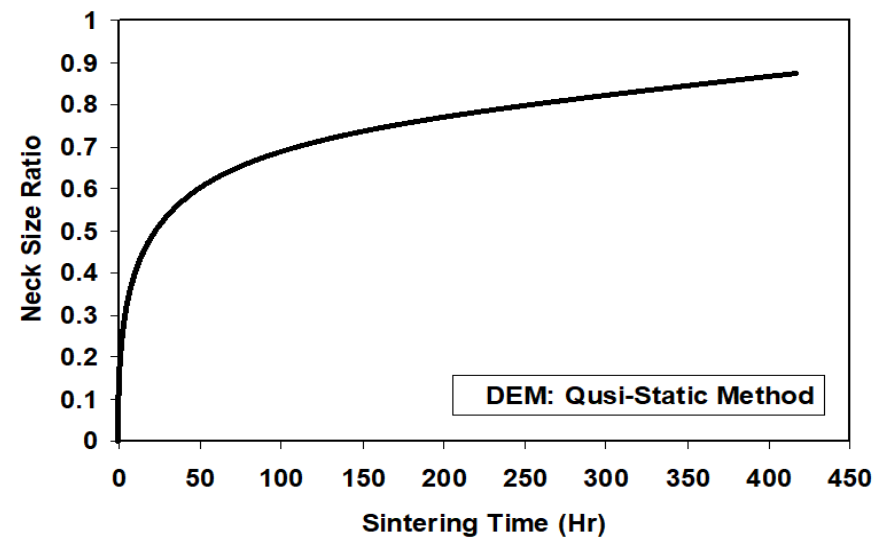

Fig. 5. Variation of neck size ratio versus sintering time of copper particle using DEM Qusi static method

in focusing on another parameter for instance shrinkage, this parameter is normally defined as the ratio of linear dimensional change to the original dimensions, in mathematical expression the change in initial length to final sintered length, $\left(\Delta L / L_{o}\right)$. The distance measured from the centre of one particle to the centre of another particle with respect to original distance between centres represents sintering shrinkage, which occurs when overlap of particles are happened. Fig. 6 shows the shrinkage ratio as a function of the sintering time for sintering process between two copper powders. The results obtained from numerical model were represented by Qusi-static method of discrete element method. Conclusion extracted from this figure shown that the shrinkage 
ratio increases with increasing sintering time, which maximum ratio was records 0.44 at sintering time $430 \mathrm{hr}$. Also, it seen that the shrinkage is proportional to the diffusion coefficient, but inversely proportional to the energy ratio [16].

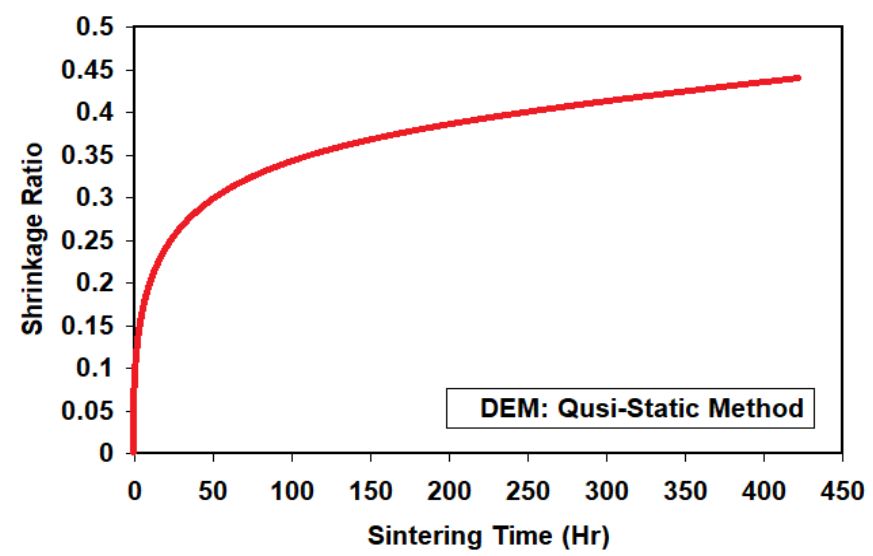

Fig. 6. Variation of shrinkage ratio versus sintering time of copper particles using DEM- Qusi-static method

During sintering process, one of the important driving forces for densification is the change in energy due to decrease or reduction in surface area [17]. In general, surface area reduction can be defined as $\Delta \mathrm{S} / \mathrm{So}$, which means that the change in the initial surface area to the final sintered surface area given as $\Delta \mathrm{S}$ to original surface area So. Based on analytical formula and numerical model, a particle coordination number of value 2, was preformed as an input parameter in computer program to determine the reduction in surface area. Fig. 8 explains the relationship between surface area reduction and neck size ratio, this relationship was carried out from numerical model of sintering process using Qusistatic method of discrete element method. It can seen from the figure that as the time increased, the reduction of surface area increase, which the later parameter become greater due to increase in neck radius to value 0.83 . Moreover, it was noted here that the percentage of surface reduction has varied between stages, means that higher value is clearly identified at initial sintering stage. In addition, increase the contact between particles results to increase surface area reduction.

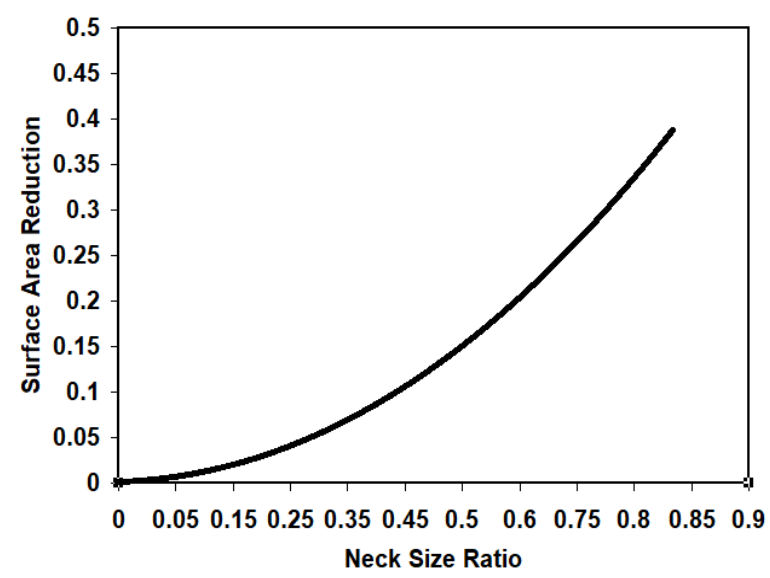

Fig. 7. The surface area reduction for copper powders

\section{EFFECTIVE PARAMETER OF SINTERING}

Particles and study their curvature's surfaces based on neck growth for different coefficient ratios using a finite difference numerical method. Generally, three parameters have been used in the repeatable simulation programs to show their effect on sintering process under different dynamic methods. The first parameter is dihedral angle as shown in Fig. 9, which is created at any two contact powders, through interfacial energies. Fig.10 shows a comparison of three curves using different dihedral angles i.e. $130^{\circ}, 145^{\circ}$ and $160^{\circ}$ a comparison was carried out between neck size ratios versus sintering process time. It was seen that the influence of change angle has small effective on sintering process. Size of particle is the second parameter that implemented in numerical program of sintering process.

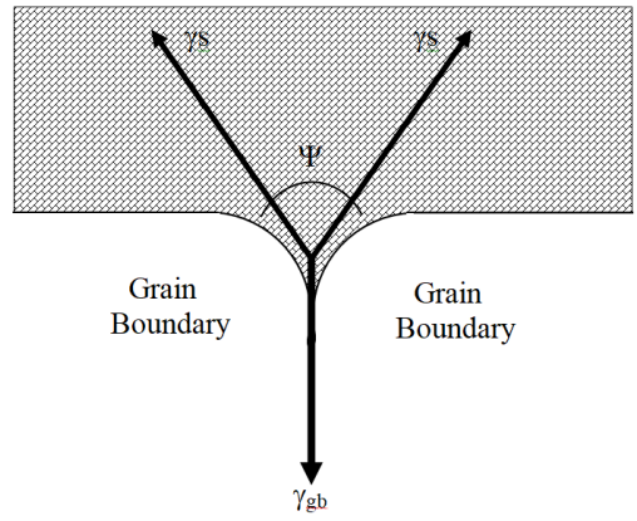

Fig. 8. The equilibrium state of dihedral angle with balance energy

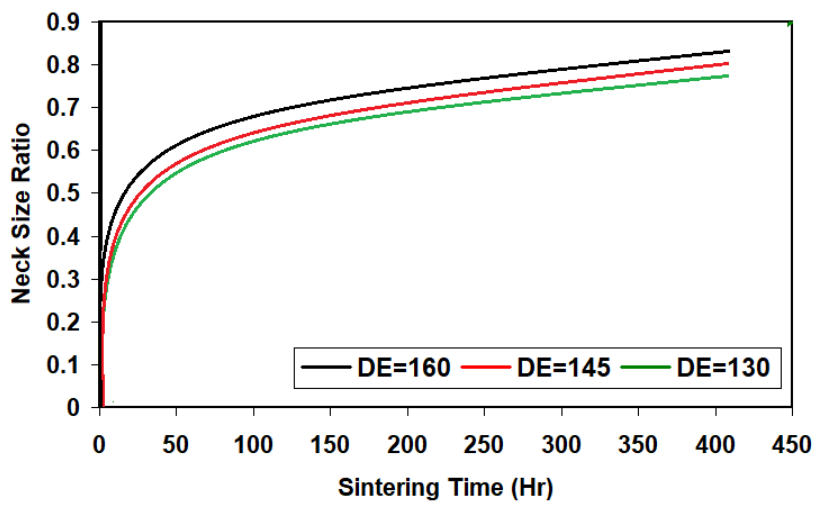

Fig. 9. Effect of dihedral angles on sintering process between neck size ratios versus time process 


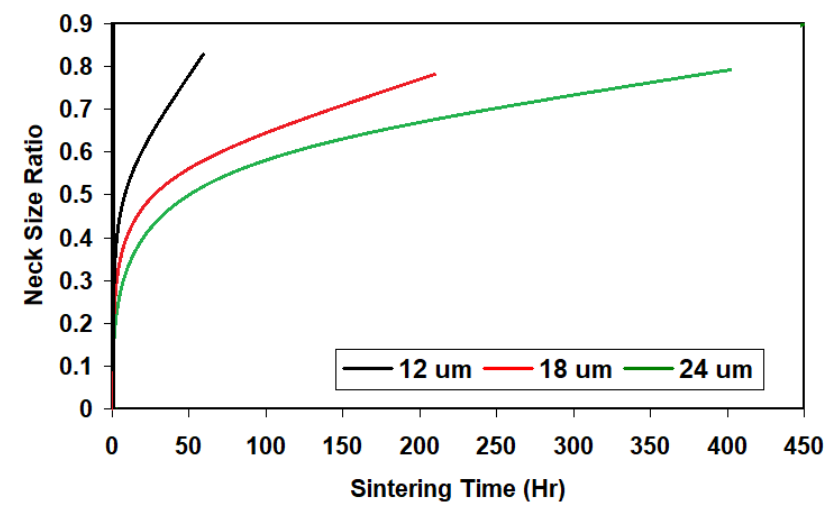

Fig. 10. Effect of particle sizes on sintering process between neck size ratios versus time process

Fig. 11 shows a comparison of different copper particle sizes, the sizes were chosen as input data to use in simulation program with values 12,18 and $24 \mu \mathrm{m}$. After simulation, the results show clear change in particle size, which the change is carried out during increased of neck size ratio along the increase in time of sintering process. The sintering time is proportional to particles size but the process is rapidly done when the particle size is small. Another significant parameter in sintering process is temperature. Fig. 12 illustrates a comparison of curves resulting from simulation of sintering process for two contact particles.

The results of simulation process which implemented the values $(1100,1200$ and $1300 \mathrm{~K})$ describe the temperature behaviour on sintering process. It was noticed that the sintering time is inversely proportional to the temperatures, which means that the sintering process is very fast by the rise of temperature. Fig. 12 shows comparison between two different methods of discrete element model and analytical solution contributed by Coble [10].

The numerical model exhibits high efficiency with spending less time in solution than analytical one, however, the curves drawn from solution are very close in behaviour; in addition, the curve of Qusi-static method is higher than two curves. In this study, the result of two discrete methods were agree well with the analytical solution [10], for the neck size ratios determined by finite difference method along sintering time, which the result is clear available for the early stage of sintering while the numerical results covered all stages reaching the end of sintering process.

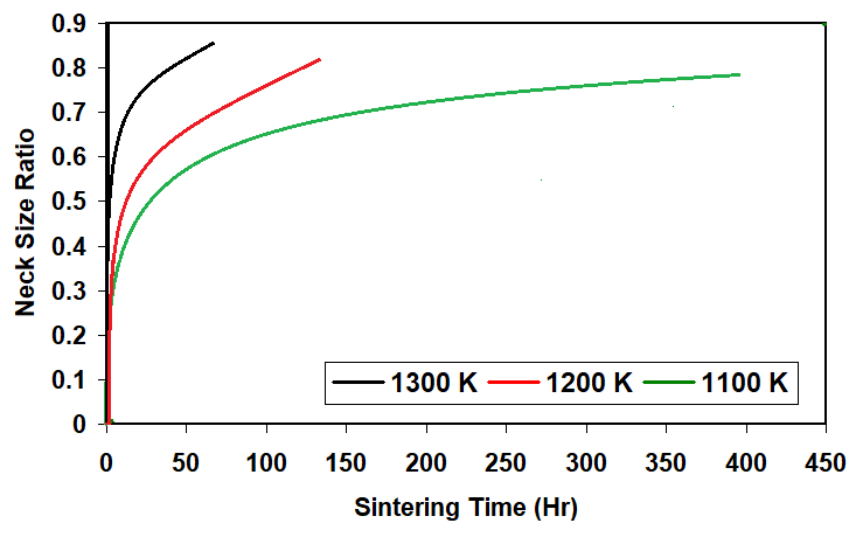

Fig. 11. Effect of temperature on sintering process between neck size ratio versus time process

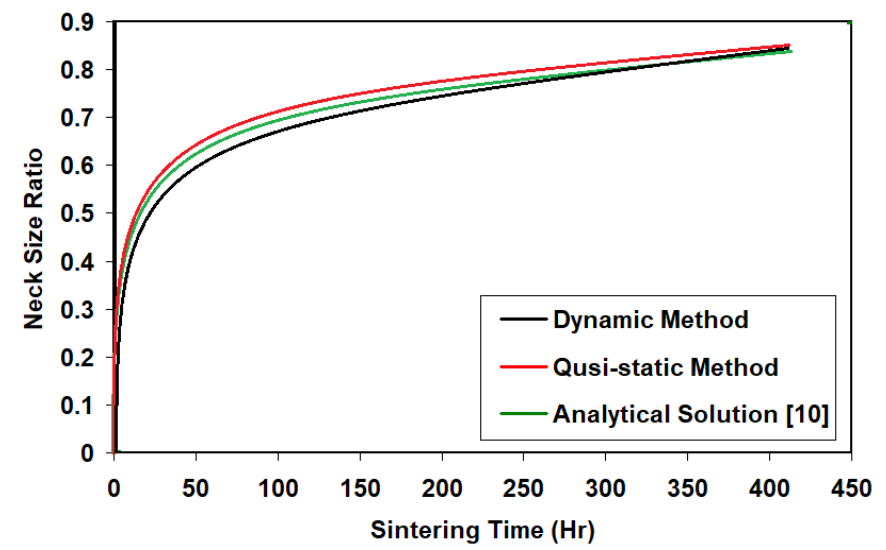

Fig. 12. Comparison between the discrete element methods (Dynamics method and Qusi-static method) with analytical solution [10]

\section{CONCLUSIONS}

The formulation of numerical models of sintering process for copper powders was carried out using two styles of discrete element method, such as, dynamics method and Qusi-static method. The two styles of discrete element methods were successfully compared using contact ratio to find the easier method of discrete element method. Three parameters are used on simulation of sintering process to study the influence of input parameters on sintering process between copper powders numerically. The following points were drawn for the solution of numerical processes such as the contact ratio (neck radius relative to powder radius) increased with increasing sintering time. At first stage of sintering process, the time consumed for completing process is very fast compared to the second and last stages. Using the two styles of discrete element method (Dynamics and Qusi-static methods) were effectively performed in simulation programs; however computer ran more easily by using Qusi-static method. Dihedral angle between contact particles has less significant effect comparing with other parameters. The time of sintering process is proportional to the particle size, which means that is increased 
due to increase in size; however the process is completely fast when the size becomes smaller. Further, the time is inversely proportional with temperature, means that the process can be done in short period due to increase in temperature. Finally, the numerical results of all input parameters show good agreement for using two different styles of DEM, and this agreement is confirmed when the numerical results were compared with the analytical one.

\section{ACKNOWLEDGEMENT}

The authors would like to say many thanks to the staff of IT lab and material lab at the University of Leicester, for their support. Also, the authors wish to thank IT lab at the University of Mosul, for providing all facilities to support and success the analytical and numerical part of this work.

\section{REFERENCES}

[1] German RM. Sintering theory and practice. Sintering Theory and Practice, by Randall M German, pp 568 ISBN 0-471-05786-X Wiley-VCH, January 1996. 1996;1.

[2] Zhang W, Schneibel J. H. The sintering of two particles by surface and grain boundary diffusion - a two-dimensional numerical study. Journal of material science. 1995; Doi 10.1016/0965-7175(95)00115-c.

[3] Parhami F, McMeeking R, Cocks A, Suo Z. A model for the sintering and coarsening of rows of spherical particles. Mechanics of Materials. 1999;31:43-61.

[4] Coble R. Initial sintering of alumina and hematite. Journal of the American Ceramic Society. 1958;41:55-62.

[5] Martin C, Bouvard D, Shima S. Study of particle rearrangement during powder compaction by the discrete element method. Journal of the Mechanics and Physics of Solids. 2003;51:667-93.
[6] Chen P, Ni J. Discrete element modeling of micro-feature hot compaction process. Transactions of the North American Manufacturing Research Institution/SME.36.

[7] Exner HE. Principles of single-phase sintering. Rev Powder Metall Phys Ceram 1,(1/4), 1979. 1979.

[8] Nosewicz S, Rojek J, Pietrzak K, Chmielewski M. Viscoelastic discrete element model of powder sintering. Powder Technology. 2013;246:15768.

[9] Yan Z, Martin CL, Guillon O, Bouvard D., Lee C.S., Microstructure

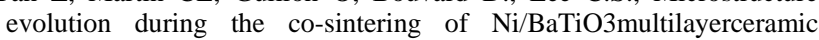
capacitors modeled by discrete element simulations. Journal of the European Ceramic Society 34 (2014) 3167-3179

[10] Coble RL. Sintering crystalline solids. I. Intermediate and final state diffusion models. Journal of applied physics. 1961;32:787-925.

[11] Coblenz W, Dynys J, Cannon R, Coble R. Initial stage solid state sintering models. A critical analysis and assessment. Sintering Processes Materials Science Research. 1980;13:141-57.

[12] Cundall. P.A. A computer model for simulating progressive, large scale movements in blocky rock systems. Proceedings of Symposium of International Society of Rock Mechanics, At Nancy, France, 1, 1971.

[13] Chen P, Ni J. Discrete element modeling of micro-feature hot compaction process. Transactions of the North American Manufacturing Research Institution/SME.36.

[14] Parhami F, McMeeking R, Cocks A, Suo Z. A model for the sintering and coarsening of rows of spherical particles. Mechanics of Materials. 1999;31:43-61.

[15] Martin C.L., Schneider L.C.R., Olmos L. and Bouvard D. Discrete element modeling of metallic powder sintering. Scripta Materialia, 55:425-428, 2006.

[16] Wakai F, Brakke K. Mechanics of sintering for coupled grain boundary and surface diffusion. Acta Materialia. 2011;59:5379-87.

[17] Kinery W.D, Bowen H.K, Uhlmann D.R. Introdcution to Ceramics (2nd Ed.) John Wiely and Sons, 1976. 